\title{
Asymmetries in the contributions of On- and Off-mechanisms to the ERG signal
}

\author{
Jan Kremers \\ University of Erlangen-Nürnberg, Erlangen, BY, Germany
}

\begin{abstract}
ERG responses were recorded to rapid-on and rapid-off L- and M-cone isolating sawtooth stimuli of different cone contrasts. In addition, the responses were recorded to simultaneous in-phase stimulation of the L- and M-cones at equal cone contrast. Linear responses to mirror imaged rapid-on and rapid-off sawtooth stimuli are also mirror imaged. By adding on- and offresponses, linear response components will cancel and nonlinearities will remain. Because nonlinearities that occur at a certain stage of visual processing will influence subsequent stages, linear response components will probably have an outer retinal origin and nonlinearities probably originate mainly in the inner (post-receptoral) retina. Keywords: electroretinograms, On- and Offresponses, L- and M-cones, asymmetries.
\end{abstract}

Received 31 January 2012; received in revised form 31 May 2012; accepted 31 May 2012. Available online 18 November 2013.

\section{Introduction}

The form of a system's response to a stimulus depends on the form of that stimulus. Similarly, the ERG response depends on the form of the visual stimulus. Originally, ERG recordings were mainly performed using flashed stimuli with a short duration. These responses are quite different from the responses to, e.g., a high frequency flicker. Similarly, the response to step increments and decrements in luminance are different.

The disadvantage of the short flash stimuli is that the responses to the increase in luminance (the "onresponse") and to the decrease in luminance (the "offresponse") are merged (Sieving, 1993; Sustar, Hawlina \& Brecelj, 2006). To be able to distinguish between the "on"- and "off"-responses either long flashed or ramped stimuli can be used. In the long flashed ERG (Horn et al., 2011; Sieving, 1993; Sustar et al., 2006) the time between the onset and the offset of the light is long enough to disentangle the responses. Ramps consist of a sudden luminance change (either rapid-on or rapid-off) and a relatively slow linear increase or decrease during the rest of the stimulus. Because the ERG response to the slow phases is small, these stimuli are suitable to isolate either "on"- or "off"-responses using rapid-on and rapid-off stimuli, respectively (Alexander, Barnes

Jan Kremers, Department of Ophthalmology, University Hospital Erlangen, Schwabachanlage 6, 91054 Erlangen, Germany. Correspondence regarding this article should be directed to: Jan Kremers, Department of Ophthalmology, University Hospital Erlangen, Schwabachanlage 6, D-91054 Erlangen, Germany. Phone: +49 91318544595 E-mail: jan. kremers@uk-erlangen.de
\& Fishman, 2003; Alexander, Fishman, Barnes \& Grover, 2001; Barnes, Alexander \& Fishman, 2002).

Although as mentioned, it can be expected that the ERG to different stimuli can be quite different, it is surprising that the responses to increments ("on"responses) and decrements ("off"-responses) in light intensities (or in photoreceptor excitations) are indeed so dissimilar that they seemingly bear no relationship with each other (Alexander et al., 2001; Rodrigues, Da Silva Filho, Silveira \& Kremers, 2010; Sustar et al., 2006; Viswanathan, Frishman \& Robson, 2000). The "on"-response shows an initial trough followed by a positive deflection that is homologue to the b-wave in the flash ERG (Frishman, 2006) and by a second trough that has about the same latency as photopic negative response (PhNR) of the flash ERG. The "off'-response often only shows a marked positive deflection: a d-wave followed by a PhNR-like negativity. These differences between the "on"- and "off"-responses most probably originate in asymmetries in the "on"- and "off"-postreceptoral pathways.

Several studies have used these stimuli to detect selective changes in the different components of the "on"- and "off"- responses caused by a retinal disorder (Alexander et al., 2003; Alexander et al., 2001; Alexander, Fishman, Peachey, Marchese \& Tso, 1992; Barnes et al., 2002; Dryja et al., 2005). However, only a few studies have concentrated upon a direct comparison between on- and off-responses by looking at symmetrical and asymmetrical components (Luo \& Frishman, 2011).

Different post-receptoral pathways may receive distinct signals from different cone types with characteristic amplitudes and relative phases. For 
example, retinal ganglion cells belonging to the parvocellular pathway receive L- and M-cone input but no S-cone input. The L- and M-cone driven signals are about equal in strength and are in counterphase at low temporal frequencies. The responses therefore display cone opponency. In contrast, cells belonging to the magnocellular pathways receive synergistic input from the L- and M-cones with the L-cone driven signal on average about twice as strong as the M-cone driven signal. This relative strength varies substantially between subjects and is proportional to the numbers of L- and M-cones in the retina (Kremers et al., 2000).

Here I present the results of measurements to rapid-on and rapid-off sawtooth stimuli to compare the "on"- and "off"-responses quantitatively and to evaluate features that reflect asymmetrical nonlinearities in the retinal processing stream leading to the net ERG signal. The stimuli were designed to either stimulate both Land $\mathrm{M}$-cone pathways in phase with equal cone contrasts or to selectively stimulate the L- and M-cones.

\section{Methods}

\section{Subjects}

The study was performed in accordance with the tenets of the Declaration of Helsinki. Six normal healthy subjects (two females aged 32 and 46 years and four males between 36 and 51 years old) participated in this study. All subjects had normal color vision and did not suffer from any retinal disorder. The subjects rested their heads on a chin rest and a forehead bar and were seated in front of the stimulus monitor with a distance to the observers' eyes of $10 \mathrm{~cm}$. The subjects were requested to fixate a cross in the middle of the screen. ERGs were recorded from one eye using DTL electrodes. Gold cup electrodes placed at the ipsilateral temple and the forehead were used as reference and ground electrodes, respectively. The other eye was covered by an eye patch. The pupils of the eyes from which the ERGs were recorded were dilated with mydriaticum (tropicamid). If requested by the subject, a topical anaesthetic (oxybuprocain) was administered.

\section{Stimuli}

The stimuli were presented on a BARCO color CRT screen (CCID $7751 \mathrm{MKII} ; 100 \mathrm{~Hz}$ frame rate) controlled by a VSG 2/2 (Cambridge Research Systems) graphics card. Four Hz sawtooth stimuli (period time: $250 \mathrm{msec}$ ) were presented. The stimuli were spatially homogeneous and subtended 124 by 108 arc deg at the $10 \mathrm{~cm}$ viewing distance. The mean luminance was $66 \mathrm{~cd} / \mathrm{m}^{2}\left(20,40\right.$ and $6 \mathrm{~cd} / \mathrm{m}^{2}$ from the red, green and blue phosphors, respectively). The mean chromaticity in the CIE 1964 large field diagram was $(0.32,0.31)$. The resultant retinal illuminance was about 3300 phot td (assuming an 8-mm pupil diameter; the Stiles-Crawford effect may decrease the effective retinal illuminance) at which rod-driven responses were strongly suppressed, although a small influence of rod activity cannot be excluded (Kremers, Czop \& Link, 2009). S-cones were not stimulated by any of the stimuli.

Three stimulus types were used: one in which the Land $\mathrm{M}$-cones were stimulated in phase and with equal stimulus strength (when expressed in cone contrasts; called $\mathrm{L}+\mathrm{M}$ ); a second in which only the L-cones were stimulated; and a third stimulus that stimulated selectively the M-cones. The maximal possible stimulus strength resulted in $76.1 \%$ cone contrast (in each cone) for the combined L+M-cone stimuli, 29.0\% $\mathrm{M}$-cone contrast for the M-cone isolating stimuli and 23.3\% L-cone contrast for the selective L-cone stimuli. ERG responses were measured at these maximal contrasts and at $0.75,0.5$ and 0.25 times the maximal contrast.

\section{Procedure}

The signals were amplified 100,000 times and bandpass filtered with 3 and $300 \mathrm{~Hz}$ cut-off frequencies by a Grass amplifier. The signals were sampled at $2 \mathrm{KHz}$ by a CED1401 on-line computer. Data acquisition was triggered by TTL pulses generated by the VSG $2 / 2$ board. The subjects were light adapted to room illumination for at least $10 \mathrm{~min}$ prior to the measurements. To avoid stimulus-onset artifacts, the first $4 \mathrm{~s}$ in the recordings were discarded. Each recording lasted $48 \mathrm{~s}$. The subjects were requested to suppress blinks and eye movements during the measurements. None of the measurements were rejected even if blinks appeared during the measurements. Between the measurements at different stimulus contrasts for a particular condition the subjects were allowed to blink several times. Between the measurements at different cone stimulus conditions the subjects could pause as long as they wished. However, the subjects never required breaks longer than about $10 \mathrm{~s}$.

\section{Data analysis}

The signal processing pathways that lead to an ERG response may include several nonlinearities. Once nonlinearity occurs, all subsequent processes are affected by this nonlinearity. Therefore, linear processes are expected to be visible in the ERG only when they occur early in the visual processing pathway. One of the properties of linear processes, and of simple contrast dependent nonlinearities, is that the response to an inverted stimulus is also inverted. This linear property can be derived from the definition of the linear system in which the response to the addition of two stimuli equals the addition to the separate stimuli: $R\left(S_{1}+S_{2}\right)=R\left(S_{1}\right)+R\left(S_{2}\right)$. As a consequence, the responses to a stimulus and its inverted stimulus cancel out when they are added: $R\left(S_{1}\right)+R\left(-S_{1}\right)=R(0)=0$. As a result, when the responses to the two sawtooth stimuli of opposite polarity are added, any residual response reveals asymmetrical nonlinearities in the ERG response, most probably with post-receptoral origins (Luo \& Frishman, 2011; Rodrigues et al., 2010). 


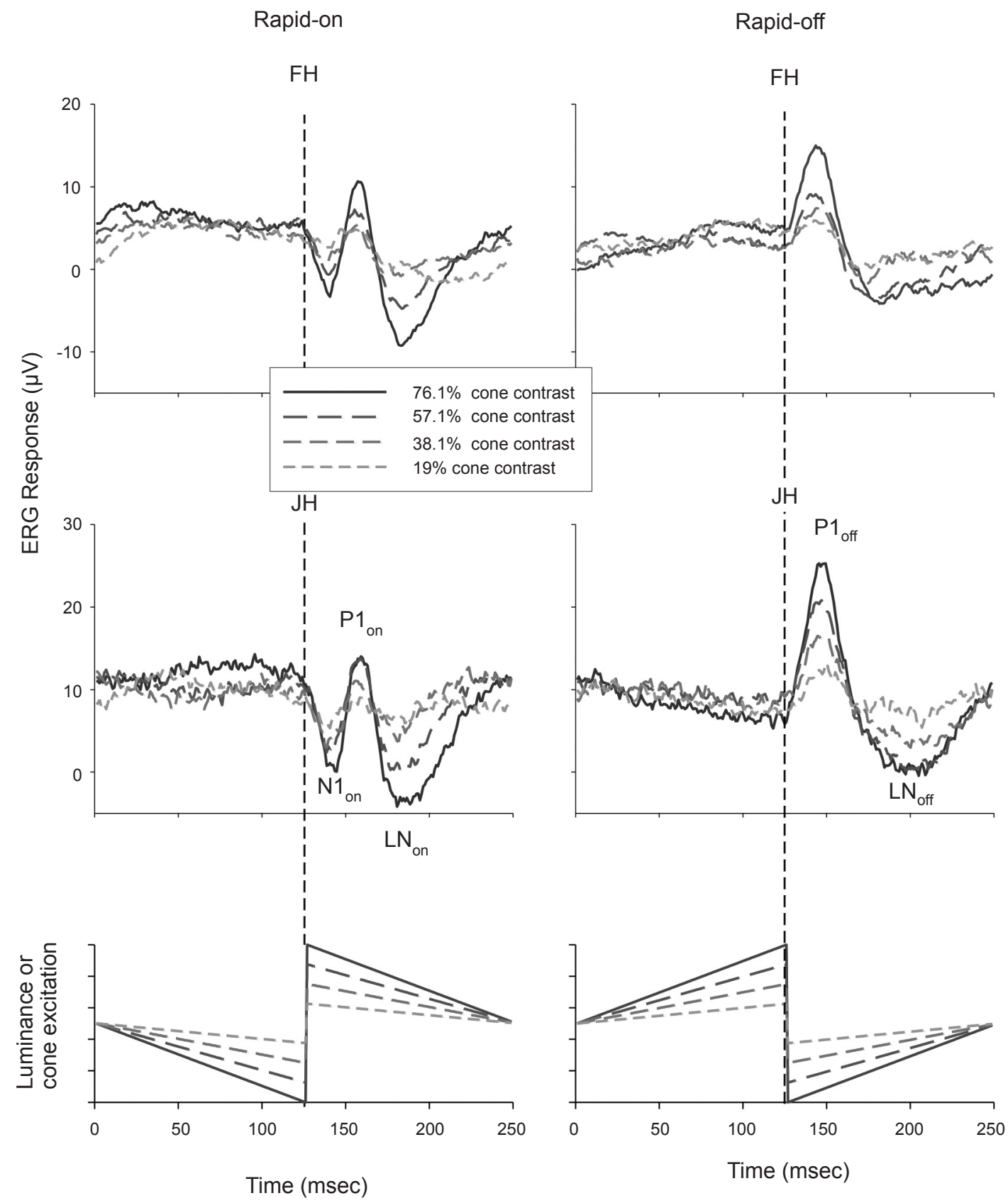

Figure 1: ERG Responses measured in two subjects to rapid-on (left plots) and rapid-off (right plots) sawtooth stimuli. The S-cone excitation was not modulated by this stimulus. L- and M-cones were modulated simultaneously in phase and with equal cone contrast. The responses to the rapid-on sawtooth stimuli display an (a-wave-like) negative $\mathrm{N} 1_{\text {on }}$ trough followed by a positive (b-wave-like) $\mathrm{P} 1_{\text {on }}$ peak and a second late negative trough $\left(\mathrm{LN}_{\mathrm{on}}\right)$. The response to the rapid-off sawtooth stimulus clearly shows a positive d-wave $\left(\mathrm{P} 1_{\text {off }}\right)$ followed by trough $\left(\mathrm{LN}_{\mathrm{off}}\right)$. The dashed line indicates the time of rapid excitation transition. The first responses to the rapid transitions in the stimulus appear with only a few milliseconds delay.

\section{Results}

Figure 1 displays the averaged ERG responses to simultaneous in-phase sawtooth modulation of L- and M-cones presented at four different contrasts for two different subjects. Similar responses were obtained in the other subjects. In the left plots, the responses to rapid-on sawtooth modulation are presented, whereas the right plots show the responses to rapid-off ramps. Data are presented in such a manner that the rapid transitions occur in the middle of the plot (at 125 msec indicated by the dashed lines). The responses resemble those of sawtooth luminance modulation presented previously (Alexander et al., 2003; Alexander et al., 2001; Barnes et al., 2002): rapid-on transitions elicit responses that contain a negative component $\left(\mathrm{N} 1_{\text {on }}\right.$; comparable to the a-wave in the flash ERG) with an implicit time of about $20 \mathrm{msec}$ followed by a positive b-wave-like peak ( $\mathrm{P} 1_{\text {on }}$; delay time about $40 \mathrm{msec}$ ) and a second (late) negative component ( $\mathrm{LN}_{\text {on }}$ about $70 \mathrm{msec}$ after the rapid change; homologue to the PhNR). A single positive peak with a 
time to peak of about $25 \mathrm{msec}\left(\mathrm{P} 1_{\text {off }}\right.$ homologue to the d-wave; right plots) can be observed after the rapid-off transition. After the $\mathrm{P}_{\text {off, }}$, another trough, the $\mathrm{LN}_{\text {off }}$, is present.

Figure 1 clearly shows that the responses to the rapid transitions in the rapid-on and rapid-off sawtooth stimuli are not mirror images of each other, indicating an asymmetry between on- and off-ERG responses. As mentioned in the Methods section, mirror-imaged responses are expected when the system leading to an ERG response is linear or involves simple contrast dependent nonlinearities (such as saturation or thresholds) that influence the on- and off-responses in an identical manner.

To study which ERG components are symmetrical (involving linear and simple contrast-dependent nonlinear mechanisms) and which are asymmetrical, the ERG responses to rapid-on and rapid-off stimuli were added.

In Figure 2 the response-additions are displayed for the six subjects. A positive peak (called $\mathrm{P} 1_{\text {add }}$ ) occurs at approximately $25 \mathrm{msec}$ after the rapid transition followed by a negative deflection $\left(\mathrm{LN}_{\text {add }}\right)$ about 50 msec after the rapid transition. The $\mathrm{N} 1_{\text {on }}$ component of the rapid-on responses partially cancelled the $\mathrm{P} 1_{\text {off }}$ responses, indicating that the cancelled responses are mirror images of each other. This strongly suggests that these components have identical origins (probably early in the processing pathway) and originate in linear and possibly contrast-dependent nonlinear mechanisms.

The addition of the responses reveals features that do not cancel and reflect asymmetries between the responses to rapid-on and rapid-off ramps. I propose that these asymmetries involve differences between on- and off-pathways that most probably have post-receptoral origins (such as different numbers of on- and off-bipolar cells or rectifying nonlinearities, e.g., in the spikegenerating mechanisms of the retinal ganglion cells; see Discussion). Moreover, the latencies of the $\mathrm{P} 1_{\text {add }}$ and the $\mathrm{LN}_{\text {add }}$ correspond to those of the b-wave and the $\mathrm{PhNR}$ in the full field flash ERG and may have similar origins [possibly in the bipolar and retinal ganglion cells, respectively (Bush \& Sieving, 1996; Viswanathan et al., 2000; Viswanathan, Frishman, Robson, Harwerth \& Smith Iii, 1999; Viswanathan, Frishman, Robson \& Walters, 2001)].

Figure 3 displays the rapid-on and rapid-off responses of the same two subjects whose responses were displayed in Figure 1 using L- and M-cone isolating stimuli. The responses are noisier than in the $\mathrm{L}+\mathrm{M}$-cone condition because the maximal possible cone contrasts $(23.3 \%$ L-cone contrast; $29.0 \%$ M-cone contrast) are much smaller in the cone-isolating stimuli. Moreover, the numbers of cones that are stimulated are smaller in the cone-isolating conditions in comparison with the L+M-condition.

In addition to the differences in response amplitude, particularly the responses to M-cone-isolating stimuli have a different waveform than the responses to $\mathrm{L}+\mathrm{M}$ and to L-cone isolating stimuli. In the responses to the M-cone isolating rapid-on sawtooth stimuli an N1 is lacking. However, a small N1 can be found in the offresponse. In summary, the $\mathrm{M}$-on response resembles the L-off response and the M-off response resembles the L-on response. We also recently observed this in responses to M-cone isolating on- and off-responses using four primary LED stimulators (McKeefry et al. (2012) ARVO E-abstract 5710; Kremers et al. (2012) ARVO E-abstract 775).

On the other hand, the L+M-stimulus lacks a red-green component and thus mainly stimulates only two post-receptoral retinogeniculate pathways (the magnocellular pathway and the blue-sensitive koniocellular pathway). The cone isolating condition additionally stimulates the parvocellular pathway. Although the pathway leading to an ERG signal is not identical to the retinogeniculate pathways, there is evidence that there is a causal relationship between the flicker ERG and the activity in the parvo- and magnocellular pathways (Kremers \& Link, 2008; Kremers, Rodrigues, Silveira \& da Silva-Filho, 2010).

In Figure 4, the summation of the rapid-on and rapidoff responses to L- (Figure 4a) and M-cone isolating stimuli (Figure 4b) is shown, revealing the asymmetries in the responses. All subjects displayed only a small positive $\mathrm{P} 1_{\text {add }}$ peak in the L-cone isolating conditions, whereas for about half of the subjects a positive peak was found in the M-cone isolating conditions. The Land $\mathrm{M}$-cone isolating stimuli elicited $\mathrm{LN}_{\text {add }}$ responses that had similar amplitudes. This indicates that the $\mathrm{LN}_{\text {add }}$ responses do not depend on the number of cones present in the retina but seem to reflect post-receptoral processing that compensates for cone numbers.

To quantify the response amplitudes in the additions, I determined the difference between the maximal potential between 0 and $46 \mathrm{msec}$ after the rapid transition for the $\mathrm{P} 1_{\text {add }}$ and the minimal $\mathrm{LN}_{\text {add }}$ potential occurring between 0 and $125 \mathrm{msec}$ after the rapid transition for all conditions and contrasts. The mean amplitudes $( \pm$ s.d.) are displayed in Figure 5 as a function of cone contrast. It can be seen that the amplitude increases with increasing cone contrast. Furthermore, at similar cone contrasts the amplitudes in the three stimulus conditions are similar. I calculated individual L-/M-ratios from the ERG amplitudes using L- and M-cone isolating conditions with similar cone contrasts $(23.3 \% \mathrm{~L}-21.7 \% \mathrm{M}$-cone contrast and $17.5 \%$ L- $14.5 \% \mathrm{M}$-cone contrast). From the individual L-/Mratios the mean ratios were calculated (by calculating the logarithms of individual values before averaging the data and converting them back into a linear scale; the reason for this is that a ratio is not normally distributed, whereas its logarithm is). The mean L-/M-ratio was 1.15 for the $23.3 \% \mathrm{~L}-/ 21.7 \% \mathrm{M}$-cone contrasts and 1.23 for the for the $17.5 \% \mathrm{~L}-/ 14.5 \% \mathrm{M}$-cone contrast.

It was previously found that performing the cone isolating modulations at high temporal frequency most often resulted in larger L-cone driven responses than 

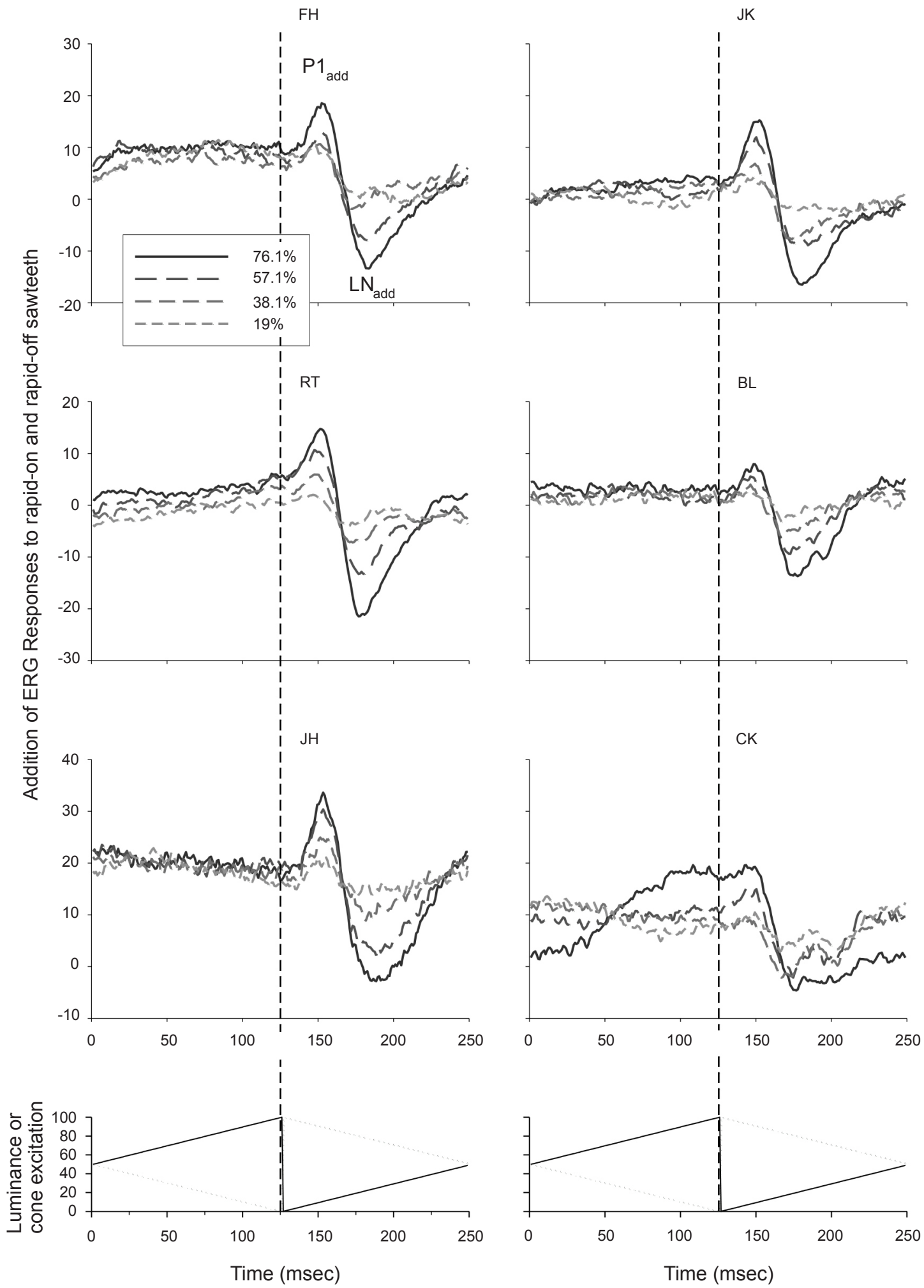

Figure 2: The addition of the responses to rapid-on and rapid-off sawtooth stimuli for the two subjects, whose responses are shown in Fig. 1, plus additional data from four other subjects. The $\mathrm{N} 1_{\text {on }}$ component is partially cancelled by the $\mathrm{P} 1_{\text {off }}$ response to the rapid-off sawtooth stimulus because the initial negative component is lacking, strongly suggesting that the cancelled response components are mainly linear, or only involve simple contrast dependent nonlinearities, and that they have similar cellular origins, probably early in the retinal processing pathway. The late trough $\left(\mathrm{LN}_{\text {add }}\right)$ is related to the two $\mathrm{LN}$ components in the responses to rapid-on and rapid-off sawtooth stimuli. 


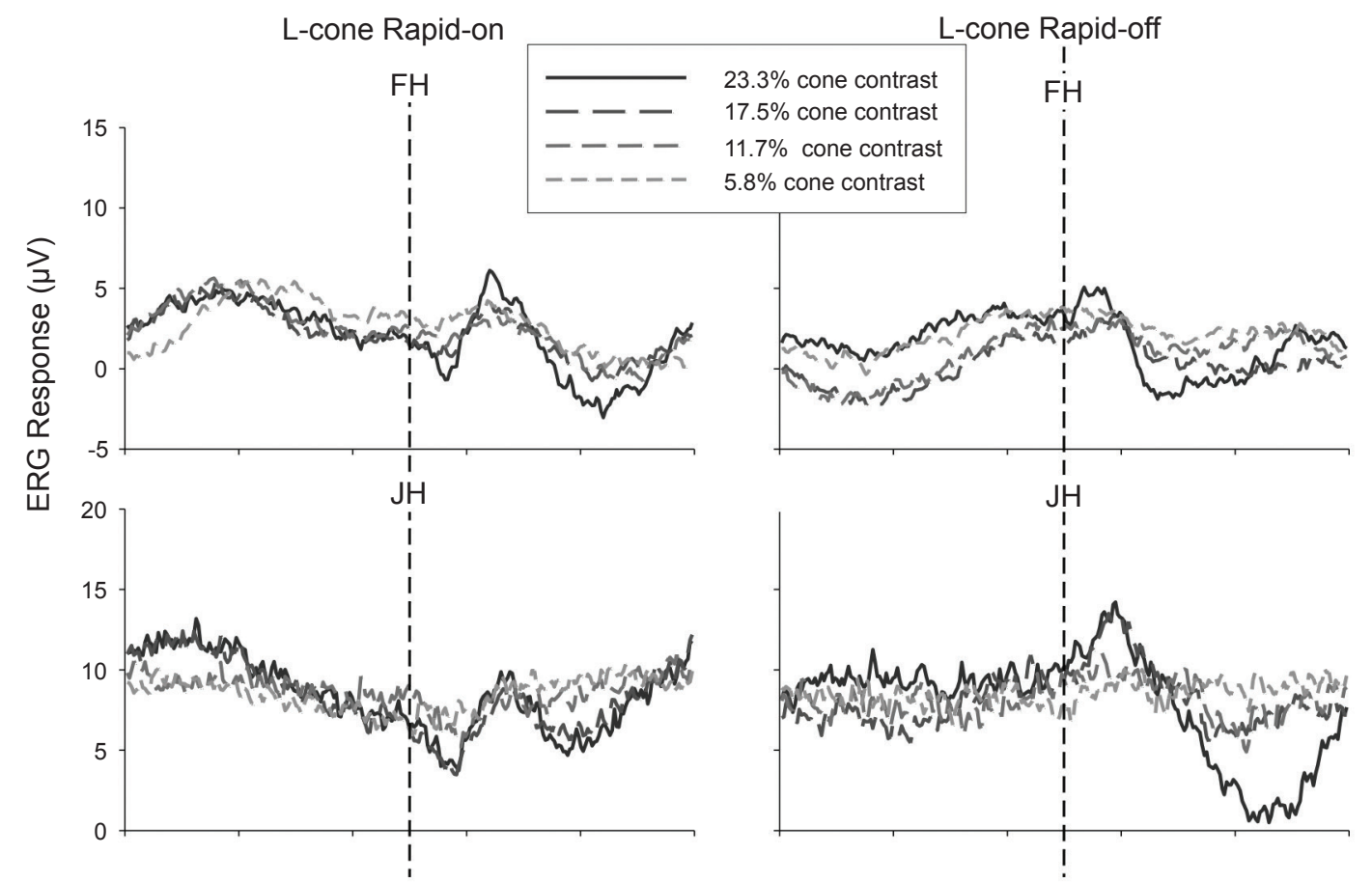

M-cone Rapid-on

M-cone Rapid-off

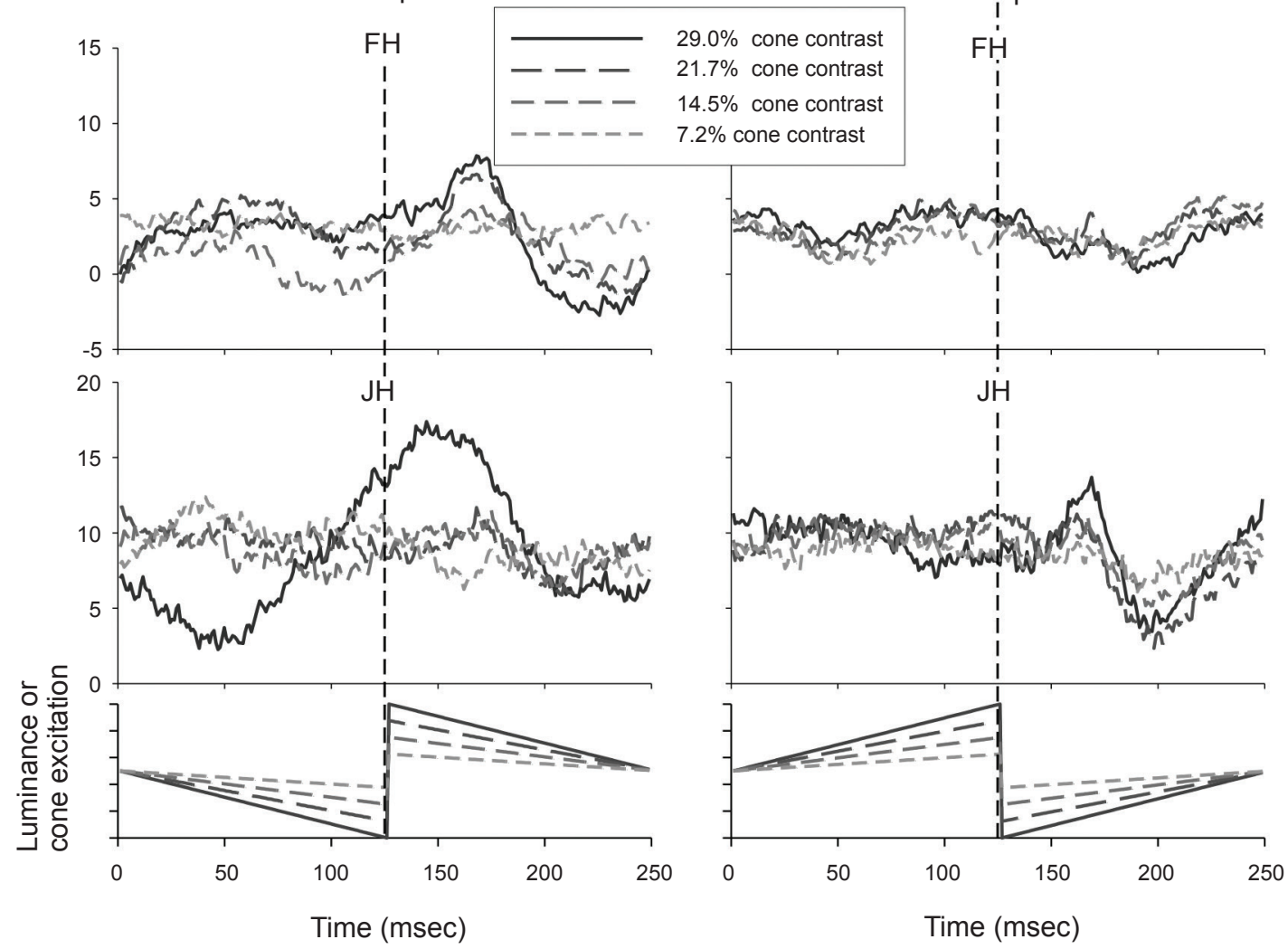

Figure 3: L-cone driven (upper four pots) and M-cone driven (lower four plots) ERG responses measured in two subjects. The left panels display the responses to rapid-on sawtooth stimuli. The right panels show the responses to the rapid-off sawtooth stimuli. 

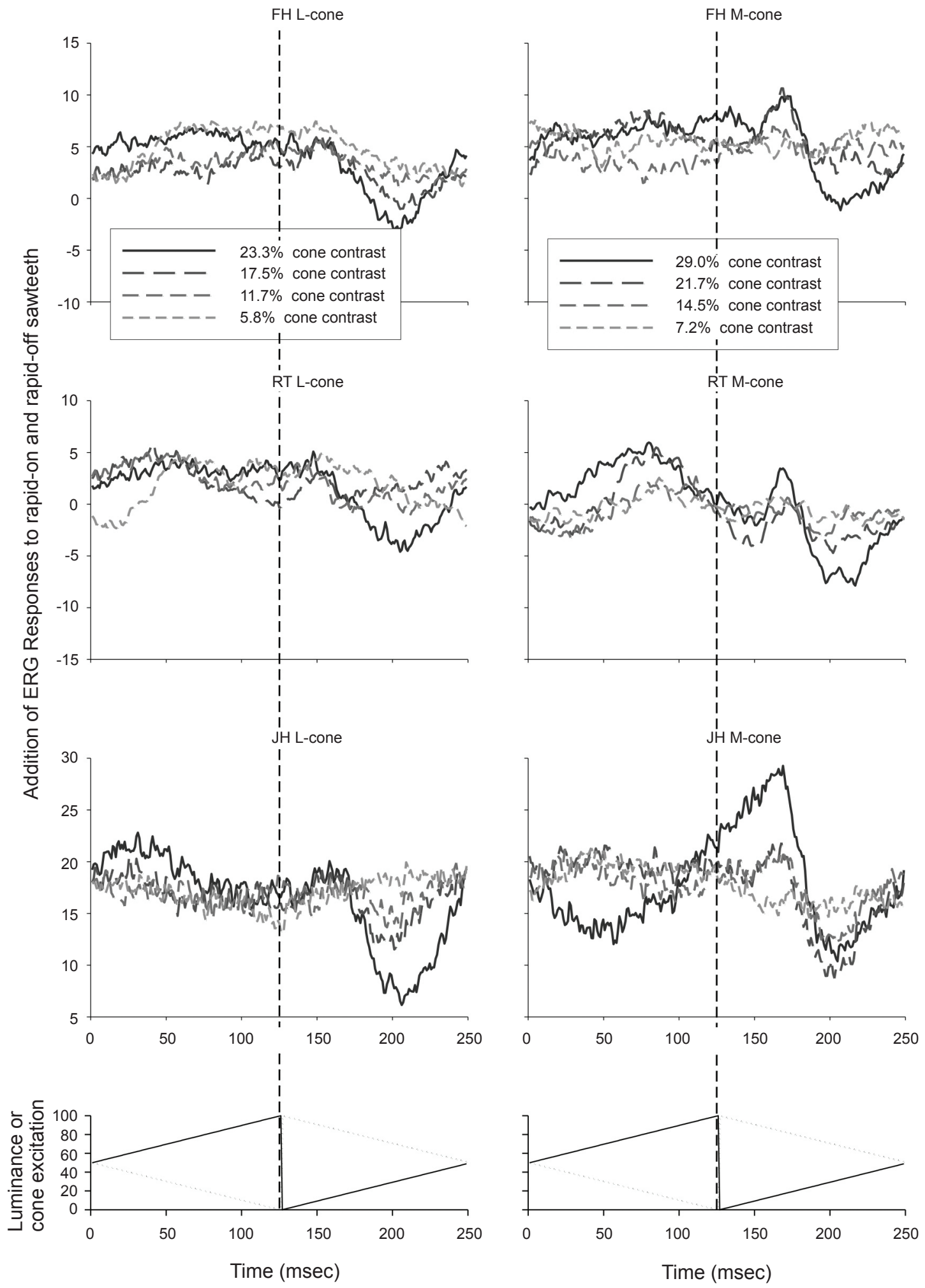

Figure 4a: Results of addition of the responses to rapid-on and rapid-off sawtooth stimuli for L- and M-cone isolating stimuli for the two subjects whose data are shown in Fig. 3, plus comparable data from four additional subjects. The responses are small but particularly an $\mathrm{LN}_{\text {add }}$ is present in most cases. 

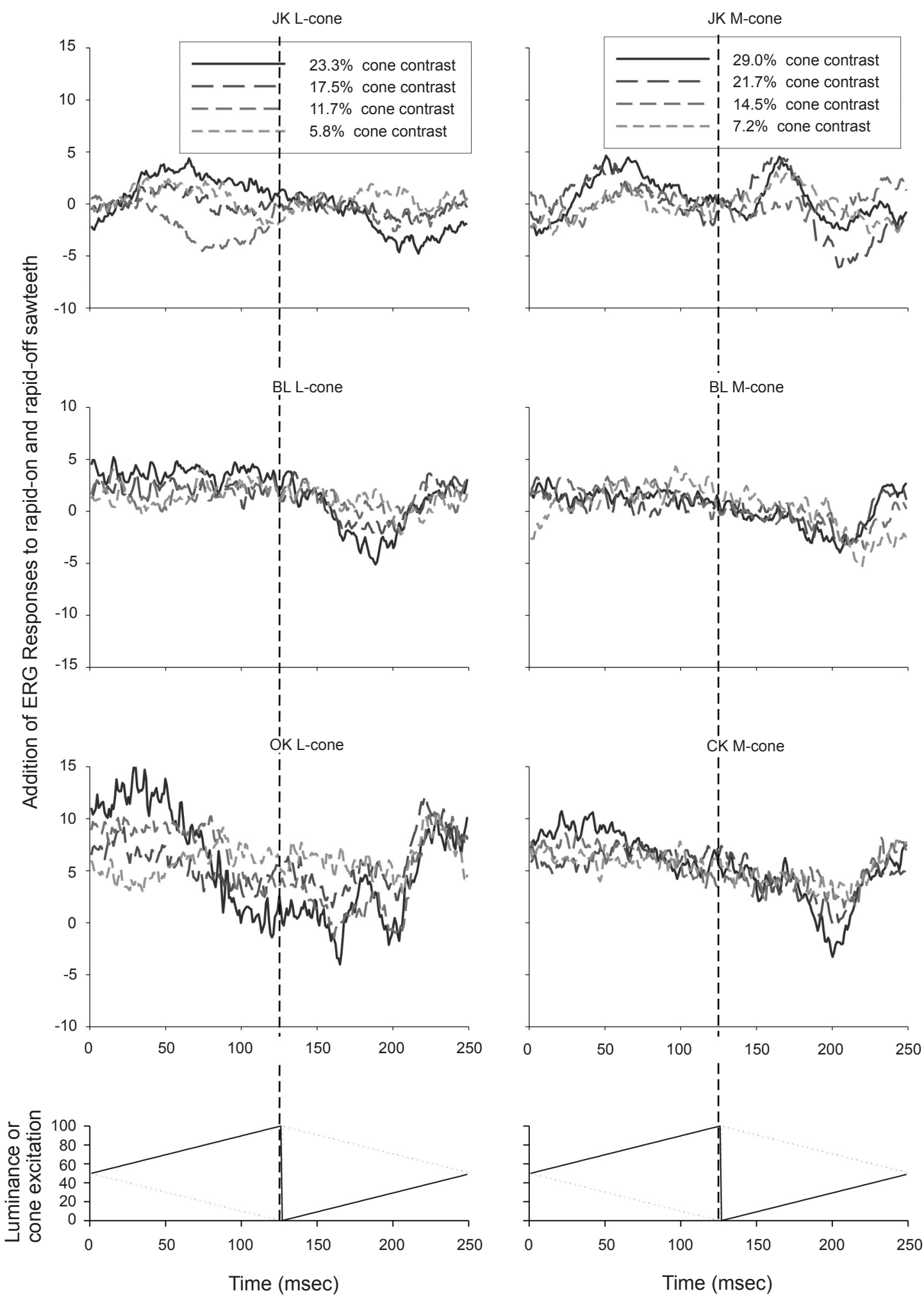

Figure 4b: Results of addition of the responses to rapid-on and rapid-off sawtooth stimuli for L- and M-cone isolating stimuli for the two subjects whose data are shown in Fig. 3, plus comparable data from four additional subjects. The responses are small but particularly an LNadd is present in most cases. 


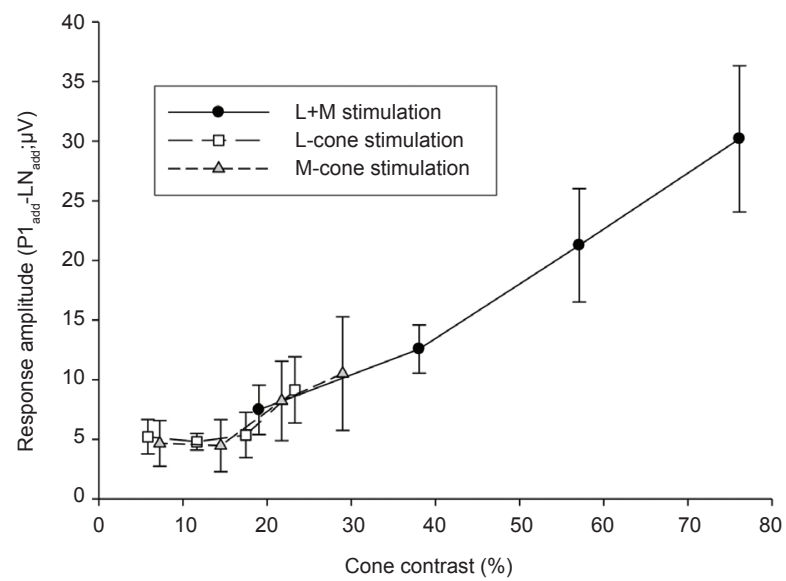

Figure 5: The mean ( \pm s.d.) $\mathrm{P} 1_{\text {add }}-\mathrm{LN}_{\text {add }}$ amplitudes measured from the added responses shown in Figs. 2 and 4 as a function of cone contrast. Cone contrast has similar influence on all amplitudes although, in the cone isolating conditions, fewer cones are stimulated than in the conditions in which the two are stimulated simultaneously. Furthermore, the amplitudes in the L- and M-cone isolating conditions are similar although most subjects have fewer M- than L-cones. This suggests that cone number does not have an effect on the $\mathrm{P} 1_{\text {add }}-\mathrm{LN}_{\text {add }}$ amplitude.

$\mathrm{M}$-cone driven responses. The ratio between $\mathrm{L}$ - and M-cone driven responses at $30 \mathrm{~Hz}$ was found to be proportional to the ratio of $\mathrm{L}$ - to $\mathrm{M}$-cone numbers in the retina (Brainard et al., 2000; Kremers et al., 2000). In four subjects (JK, BL, FH and RT) the measurements with $30 \mathrm{~Hz}$ modulation were performed (data not shown). For all four subjects, the L-cone driven response was larger than the $\mathrm{M}$-cone driven response in these conditions with L-/M-ratios varying between 2.3 and 5.0. The mean L-/M-ratio with the sawtooth stimuli in these subjects was 1.26 and 1.35 for the higher and lower contrast combinations, respectively. Therefore, it seems that the ERG responses to L- and M-cone isolating sawtooth stimuli are determined by a mechanism that compensates partially for unequal numbers of cones.

The implicit times of the $\mathrm{P}_{\text {add }}$ and the $\mathrm{LN}_{\text {add }}$ components for those conditions in which amplitudes above noise level (defined as a $\mathrm{P} 1_{\text {add }}-\mathrm{LN}_{\text {add }}$ response amplitude of $5 \mu \mathrm{V}$ ) could be obtained are displayed in Figures $6 \mathrm{~A}$ and $6 \mathrm{~B}$, respectively. The implicit times in the $\mathrm{M}$-cone isolating conditions were longer than those obtained with the L-cone isolating and the combined stimuli, suggesting that different ERG generating mechanisms are involved in these conditions. The interindividual differences are large and only a limited number of subjects, but a paired t-test shows a moderate significant difference for the implicit time of $\mathrm{P}_{\text {add }}(\mathrm{p}=0.012)$.

\section{Discussion}

In the current paper the results of ERG measurements are presented using rapid-on and rapidoff sawtooth stimuli that strongly activate On- or Offretinal pathways at the time of the rapid transition of
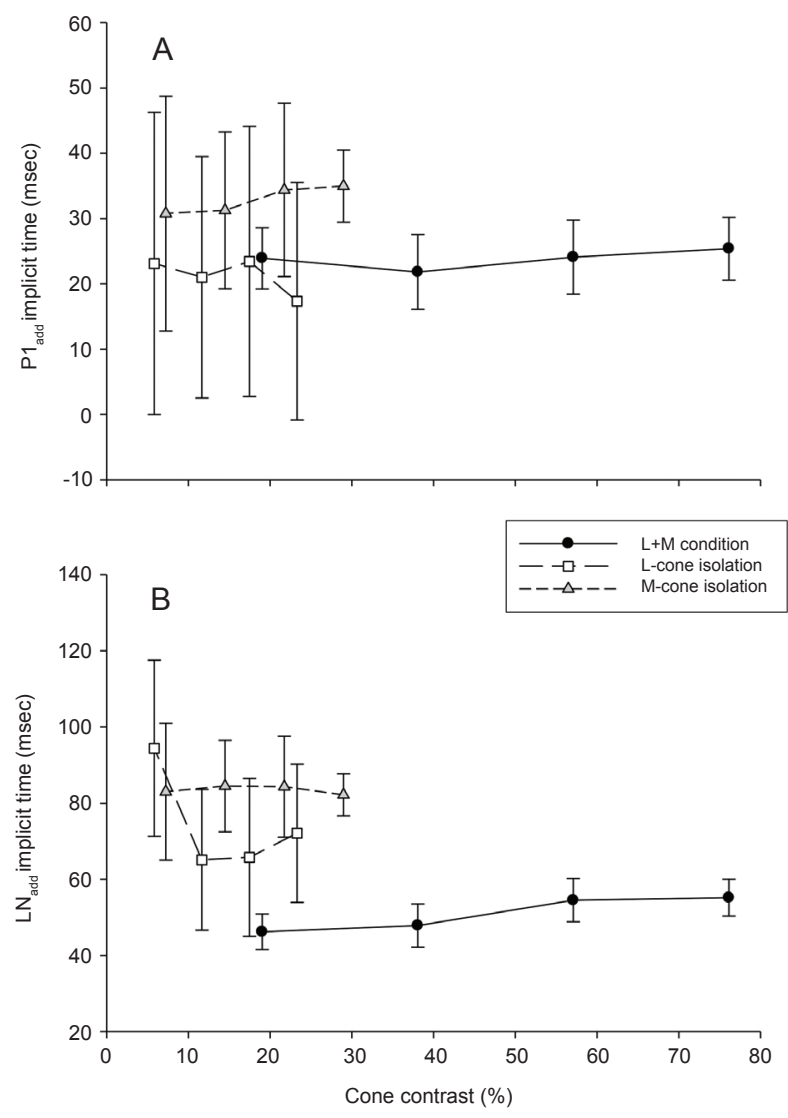

Figure 6: A: Mean $\mathrm{P} 1_{\text {add }}$ implicit times ( \pm s.d. $)$ as a function of cone contrast for the $\mathrm{L}+\mathrm{M}$ stimulus condition and the $\mathrm{M}$ and L-cone isolating conditions. With the L-cone isolating stimulus, a P1 was often not present. The implicit times were only measured for those responses in which a P1 component could be identified. Because the responses were generally smaller and noisier in the cone isolating conditions, the variability is larger resulting in larger error bars. Cone contrast has no clear influence on the timing of the response. The time to peak (implicit time) is larger in the M-cone isolating condition when compared with the L-cone condition and with the combined $\mathrm{L}+\mathrm{M}$ stimulation. B: Mean $\mathrm{LN}_{\text {add }}$ implicit times ( \pm s.d.) as a function of cone contrast for the $\mathrm{L}+\mathrm{M}$ stimulus and for the two cone isolating stimulus conditions. The $\mathrm{LN}_{\text {add }}$ component is delayed in the cone isolating conditions relative to the one in the responses to simultaneous stimulus conditions. As with the $\mathrm{P} 1_{\text {add }}$ data, the implicit times are larger in the M-cone isolating condition than in the L-cone isolating condition. The responses in the cone isolating conditions are noisier resulting in larger standard deviations.

the sawtooth (Alexander et al., 2003; Alexander et al., 1992). The stimuli were designed to isolate the L- or $\mathrm{M}$-cone responses or modulate the excitation of the two simultaneously with equal phase and contrast. None of the stimuli modulated S-cone excitation (silent S-cone substitution). A CRT screen has only three primaries, so that rod contrast cannot be controlled. To obtain full control over all photoreceptor excitations, a stimulator with four or more primaries is necessary (Pokorny, Smithson \& Quinlan, 2004; Shapiro, Pokorny \& Smith, 1996). In the present paper I assume that contributions of rod driven signals are small, although possibly owing 
to the abundance of rod photoreceptors, they cannot be completely excluded (Kremers et al., 2009).

The ERG responses to the rapid-on and rapid-off sawtooth stimuli were added so that any linear and simple contrast-dependent nonlinear responses (such as saturation) would cancel because they have the property that their responses to inverted stimuli are also inverted.

Figure 2 shows that the response addition does not display an initial negativity, strongly suggesting that a large part of the $\mathrm{P} 1_{\text {off }}$ has the same cellular origin as the $\mathrm{N} 1_{\text {on }}$-wave probably early in the signal processing cascade. These components are probably homologues of the d-and a-wave in the longflash ERG. Indeed, these components probably originate in the photoreceptors and the hyperpolarizing (off-) bipolar cells (Frishman, 2006).

The response addition can be used to extract asymmetries in the responses. The asymmetries will become more prominent the more nonlinear processes are included in the pathway leading to the ERG response. These asymmetries therefore will probably have largely post-receptoral origins. It is difficult to speculate which mechanisms may cause the measured asymmetries. One factor contributing to the asymmetries may be anatomical in origin: different numbers of "on"- and "off"-center bipolar and retinal ganglion cells may result in differences between "on"- and "off"-responses. Another mechanism may be the effects of intrinsic physiological rectifying nonlinearities.

The $\mathrm{LN}_{\text {add }}$ trough in the asymmetric response (Figures 2 and 4) is probably related to the photopic negative response (PhNR) of the flash ERG, which presumably originates in the spike-generating mechanism of the retinal ganglion cells (Viswanathan et al., 1999, 2000, 2001). Here an obvious rectifying nonlinearity is introduced because a neuron cannot generate negative numbers of spikes. As a result, an inhibiting stimulus can only decrease the firing rate to zero. An excitatory stimulus may result in a much larger increase in the number of action potentials so that the total number of action potentials of all (on- and off-) retinal ganglion cells increases. Such an increase would be visible both at a luminance increase (from on-cells) and a luminance decrease (from off-cells). Such rectifying nonlinearities are indeed present in the responses of retinal ganglion cells to sawtooth stimuli (Kremers, Lee, Pokorny \& Smith, 1993).

Addition of responses to rapid-on and rapidoff sawtooth stimuli probably reveals response characteristics similar to the pattern ERG. In the pattern, ERG symmetric on- and off-responses are thought to be cancelled out and asymmetries in the response, mainly with post-receptoral origins, remain (Bach \& Hoffmann, 2006; Luo \& Frishman, 2011; Viswanathan et al., 2000). One difference between the pattern ERG and the approach in the present study is that the cancellations in the pattern ERG derive from addition of simultaneous responses from different retinal areas whereas the post hoc response additions presented here derive from responses from the same retinal area measured at different times. Nevertheless, an experimental approach and analysis similar to ours, i.e., the addition of responses to stimulus onset and offset of long flashes, has been used successfully to simulate pattern ERG responses (Luo \& Frishman, 2011; Simpson \& Viswanathan, 2007; Viswanathan et al., 2000). Insofar as the pattern ERG has been successfully used to reveal alterations in temporal asymmetries in diseases such as glaucoma, it can be expected that this approach will prove useful in evaluation and characterization of disorders of the inner retina (Viswanathan et al., 2000; Viswanathan et al., 1999; Viswanathan et al., 2001). Indeed we recently found that the responses to sawtooth stimuli and their additions are altered in glaucoma patients (Pangeni, Lammer, Tornow, Horn \& Kremers, 2012).

One advantage of the present analysis in comparison with the pattern ERG is that it is less sensitive to optical blur. This is especially important for measurements in which the optical quality is inferior. One example may be ERG measurements in mice. Nowadays many ERG responses are performed on mice, either in wild type or in animal models for retinal disorders. For example, pattern ERGs have been measured to monitor progressive retinal ganglion cell dysfunction in the DBA/2J mouse model of glaucoma (Porciatti, Saleh \& Nagaraju, 2007; Saleh, Nagaraju \& Porciatti, 2007). However, pattern ERGs in mice typically have very low amplitudes and thus require large numbers of averages to be able to extract a reliable response. The analysis described in the present paper may provide an alternative for the procedure of pattern ERG recordings.

To illustrate this, in Figure 7 the responses in the left and the right eye of a mouse to a $200 \mathrm{msec}$ flash are displayed. Details on animal treatment and recording settings have been described elsewhere (Harazny, Scholz, Buder, Lausen \& Kremers, 2009). The recordings were performed with contact lens electrodes and the eyes were light adapted with a background of $25 \mathrm{~cd} / \mathrm{m}^{2}$. The flash intensity was $133 \mathrm{~cd} / \mathrm{m}^{2}$. The responses are averages of the ERG signals to six single flashes. In the lower plots, the responses in $200 \mathrm{msec}$ windows around flash on- and offset are summed. It can be seen that a transient response remains. This response has an amplitude about 40 times larger than the pattern ERG measured in mice (Porciatti et al., 2007; Saleh et al., 2007). This illustrates that averaging only a few responses (in our case six) is sufficient to obtain a reliable response with these stimulus parameters.

These data are notmeant to give a complete description of long-flash ERG responses in mice. Instead, this should illustrate that the addition of on- and off-responses can be a useful alternative for pattern ERGs in cases where the spatial resolution of the responding system is low and response amplitudes are small. Although the addition of on- and off-responses may not be identical to pattern ERG responses (because spatial interactions that may play a role in the pattern ERGs are not involved in the 

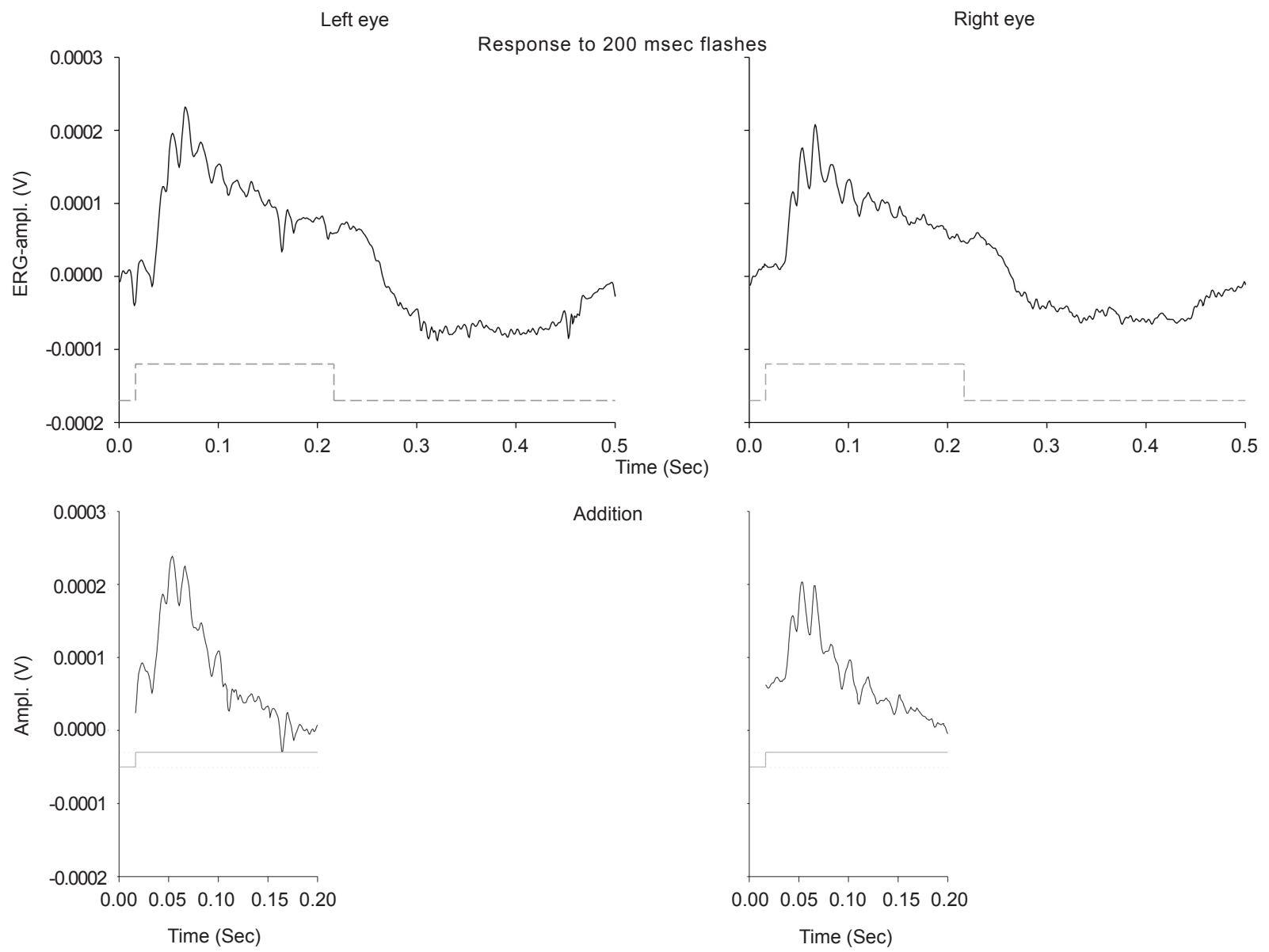

Figure 7: An example of the technique of addition of rapid-on and rapid-off responses to a $200 \mathrm{msec}$ pulse of light measured in the two eyes of a C57/BL6 mouse. Responses to six flashes were averaged (top 2 panels). In the bottom panels, the results of response addition in two $200 \mathrm{msec}$ windows (starting $17 \mathrm{msec}$ before and $183 \mathrm{msec}$ after the on- and offset of the flash) are displayed. After addition, a transient response with superimposed oscillatory potentials remains. These responses are large and can be readily measured in the mouse, where typically pattern ERG responses are very small, requiring extensive averaging to obtain a reliable signal. Stimulus conditions: background intensity was $25 \mathrm{~cd} / \mathrm{m}^{2}$; flash intensity was $133 \mathrm{~cd} / \mathrm{m}^{2}$.

full field responses), they are good approximations in non-human primates (Luo \& Frishman, 2011; Simpson \& Viswanathan, 2007; Viswanathan et al., 2000) and probably emphasize responses of the inner retina.

The comparison of the responses to the cone isolating stimuli with each other and with the conditions in which the two are modulated simultaneously reveals a few interesting features. In previous measurements, using modulation at high temporal frequency, it was found that the L-cone driven responses are generally larger than the $\mathrm{M}$-cone driven responses reflecting the larger number of L-cones (Brainard et al., 2000; Kremers, Parry, Panorgias \& Murray, 2011; Kremers et al., 2000). However, in Figures 4 and 5 it can be seen that the response amplitudes in the asymmetries to Land M-cone stimuli are similar when the cone contrasts are similar. It therefore seems that the asymmetries in the ERG responses to sawtooth stimuli involve postreceptoral mechanisms that compensate for these differences in cone number so that the net L-/M-ratio manifested in the response is about unity.
There are indications that similar compensatory mechanisms are present in the parvocellular channel (Kremers, 2003, 2011; Kremers et al., 2000; Kremers, Stepien, Scholl \& Saito, 2003). Perhaps the ERGs to sawtooth stimuli reflect some aspects of parvocellular activity. Recently we found that ERG responses to 12 $\mathrm{Hz}$ temporal modulation containing a red-green stimulus component reflect parvocellular activity (Kremers \& Link, 2008; Kremers et al., 2010). Cone opponent mechanisms are also involved in the generation of the b-wave in the full field flash ERG (Kasuga, 2001; Sperling \& Harwerth, 1971; Sperling \& Mills, 1991). In the $\mathrm{L}+\mathrm{M}$ cone condition, no red-green component is present in the stimulus and therefore the parvocellular channel is not activated in this condition so that the ERG cannot reflect a parvocellular contribution. Although the present research provides additional evidence now that activity of retinogeniculate pathways can be correlated with certain aspects of the ERG, the specific mechanisms underlying this correlation ae unclear and need more detailed examination. 


\section{Acknowledgements}

The work was supported by DFG Grant KR1317/91. JK was supported by the Neuroscience Excellence Program of the Hertie Foundation. This manuscript is dedicated to two extraordinary persons: Barry Lee, who is one of my main scientific mentors and who showed me what good science is, and Dora Fix Ventura, with whom I have had fantastic collaborations. I thank them for everything that was and for everything that will be.

\section{References}

Alexander, K.R., Barnes, C.S. \& Fishman, G.A. (2003). ON-pathway dysfunction and timing properties of the flicker ERG in carriers of X-linked retinitis pigmentosa. Investigative Ophthalmology and Visual Science, 44, 4017-4025.

Alexander, K.R., Fishman, G.A., Barnes, C.S. \& Grover, S. (2001). ON-response deficit in the electroretinogram of the cone system in X-linked retinoschisis. Investigative Ophthalmology and Visual Science, 42, 453-459.

Alexander, K.R., Fishman, G.A., Peachey, N.S., Marchese, A.L. \& Tso, M.O. (1992). 'On' response defect in paraneoplastic night blindness with cutaneous malignant melanoma. Invest.Ophthalmol Vis.Sci., 33 (3), 477-483.

Bach, M. \& Hoffmann, M. (2006). The origin of the pattern electroretinogram. In Heckenlively, J.R. \& Arden, G.B. (Eds.), Principles and Practice of Clinical Electrophysiology of Vision., 2nd edition (pp. 185-196). Cambridge, London: The MIT Press.

Barnes, C.S., Alexander, K.R. \& Fishman, G.A. (2002). A distinctive form of congenital stationary night blindness with cone ONpathway dysfunction. Ophthalmology, 109, 575-583.

Brainard, D.H., Roorda, A., Yamauchi, Y., Calderone, J.B., Metha, A.B., Neitz, M., Neitz, J., Williams, D.R. \& Jacobs, G.H. (2000). Functional consequences of the relative numbers of $\mathrm{L}$ and $\mathrm{M}$ cones. Journal of the Optical Society of America A, 17 (3), 607614.

Bush, R.A. \& Sieving, P.A. (1996). Inner retinal contributions to the primate photopic fast flicker electroretinogram. Journal of the Optical Society of America A, 13 (3), 557-565.

Dryja, T.P., McGee, T.L., Berson, E.L., Fishman, G.A., Sanberg, M.A., Alexander, K.R., ... Rajagopalan, A.S. (2005). Night blindness and abnormal cone electroretinogram $\mathrm{ON}$ responses in patients with mutations in the GRM6 gene encoding mGluR6. Proceedings National Academy of Science, 102 (12), 4884-4889.

Frishman, L.J. (2006). Origins of the electroretinogram. In Heckenlively, J.R. \& Arden, G.B. (Eds.), Principles and practice of clinical electrophysiology of vision., 2nd edition (pp. 139-183). Cambridge, London: The MIT Press.

Harazny, J., Scholz, M., Buder, T., Lausen, B. \& Kremers, J. (2009). Electrophysiological deficits in the retina of the DBA/2J mouse. Documenta Ophthalmologica.

Horn, F.K., Gottschalk, K., Mardin, C.Y., Pangeni, G., Junemann, A.G. \& Kremers, J. (2011). On and off responses of the photopic fullfield ERG in normal subjects and glaucoma patients. Doc Ophthalmol, 122 (1), 53-62.

Kasuga, T. (2001). Effect of glutamate analogues on red-green opponent interaction in monkey electroretinograms. Experimental Eye Research, 73 (3), 311-320.

Kremers, J. (2003). The assessment of L- and M-cone specific electroretinographical signals in the normal and abnormal retina. Progress in Retinal and Eye Research, 22, 579-605.

Kremers, J. (2011). Signal Pathways in the Electroretinogram. In Belušič, G. (Ed.) Electroretinograms (pp. 55-78). Rijeka: InTech.

Kremers, J., Czop, D. \& Link, B. (2009). Rod and S-cone driven ERG signals at high retinal illuminances. Documenta Ophthalmologica, $118,205-216$
Kremers, J., Lee, B.B., Pokorny, J. \& Smith, V.C. (1993). Responses of macaque ganglion cells and human observers to compound periodic waveforms. Vision Research, 33, 1997-2011.

Kremers, J. \& Link, B. (2008). Electroretinographic responses that may reflect activity of parvo- and magnocellular post-receptoral visual pathways. Journal of Vision, 8 (15/11), 1-14.

Kremers, J., Parry, N.R., Panorgias, A. \& Murray, I.J. (2011). The influence of retinal illuminance on L- and M-cone driven electroretinograms. Visual Neuroscience, 28, 129-135.

Kremers, J., Rodrigues, A.R., Silveira, L.C.L. \& da Silva-Filho, M. (2010). Flicker ERGs representing chromaticity and luminance signals. Investigative Ophthalmology \& Visual Science, 51, 577-587.

Kremers, J., Scholl, H.P.N., Knau, H., Berendschot, T.T.J.M., Usui, T. \& Sharpe, L.T. (2000). L/M cone ratios in human trichromats assesed by psychophysics, electroretinograpy, and retinal densitometry. Journal of the Optical Society of America B, 17, 517-526.

Kremers, J., Stepien, M.W., Scholl, H.P.N. \& Saito, C.A. (2003). Cone selective adaptation influences L- and M-cone driven signals in electroretinography and psychophysics. Journal of Vision, 3, 146-160.

Luo, X. \& Frishman, L.J. (2011). Retinal pathway origins of the pattern electroretinogram (PERG). Invest Ophthalmol Vis Sci, 52 (12), 8571-8584.

Pangeni, G., Lammer, R., Tornow, R.P., Horn, F.K. \& Kremers, J. (2012). On- and off-response ERGs elicited by sawtooth stimuli in normal subjects and glaucoma patients. Documenta Ophthalmologica.

Pokorny, J., Smithson, H. \& Quinlan, J. (2004). Photostimulator allowing independent control of rods and the three cone types. Visual Neuroscience, 21 (3), 263-267.

Porciatti, V., Saleh, M. \& Nagaraju, M. (2007). The pattern electroretinogram as a tool to monitor progressive retinal ganglion cell dysfunction in the DBA/2J mouse model of glaucoma. Investigative Ophthalmology \& Visual Science, 48 (2), 745-751.

Rodrigues, A.R., Da Silva Filho, M., Silveira, L.C. \& Kremers, J. (2010). Spatial distributions of on- and off-responses determined with the multifocal ERG. Documenta Ophthalmologica, 120 (2), 145-158.

Saleh, M., Nagaraju, M. \& Porciatti, V. (2007). Longitudinal evaluation of retinal ganglion cell function and IOP in the DBA/2J mouse model of glaucoma. Investigative Ophthalmology \& Visual Science, 48 (10), 4564-4572.

Shapiro, A.G., Pokorny, J. \& Smith, V.C. (1996). Cone-rod receptor spaces with illustrations that use the CRT phosphor and lightemitting-diode spectra. Journal of the Optical Society of America A, 13, 2319-2328.

Sieving, P.A. (1993). Photopic on- and off-pathway abnormalities in retinal dystrophies. Transactions of the American Ophthalmological Society, 91, 701-773.

Simpson, M. \& Viswanathan, S. (2007). Comparison of uniform field and pattern electroretinograms of humans. Journal of Modern Optics, 54, 1281-1288.

Sperling, H.G. \& Harwerth, R.S. (1971). Red-green cone interaction in the increment-threshold spectral sensitivity of primates. Science, $172,180-184$.

Sperling, H.G. \& Mills, S.L. (1991). Red-green interactions in the spectral sensitivity of primates as derived from ERG and behavioral data. Visual Neuroscience, 7, 75-86.

Sustar, M., Hawlina, M. \& Brecelj, J. (2006). ON- and OFFresponse of the photopic electroretinogram in relation to stimulus characteristics. Documenta Ophthalmologica, 113, 43-52.

Viswanathan, S., Frishman, L.J. \& Robson, J.G. (2000). The uniform field and pattern ERG in macaques with experimental glaucoma: Removal of spiking activity. Investigative Ophthalmology \& Visual Science, 41 (9), 2797-2810.

Viswanathan, S., Frishman, L.J., Robson, J.G., Harwerth, R.S. \& Smith III, E.L. (1999). The photopic negative response of the macaque electroretinogram: reduction by experimental glaucoma. Investigative Ophthalmology \& Visual Science, 40 (6), 1124-1136. Viswanathan, S., Frishman, L.J., Robson, J.G. \& Walters, J.W. (2001). The photopic negative response of the flash electroretinogram in primary open angle glaucoma. Investigative Ophthalmology \& Visual Science, 42 (2), 514-522. 